\title{
O pombo (Columba livia) como agente carreador de Salmonella spp. e as implicações em saúde pública
}

\section{The pigeon (Columba livia) as a carrier agent of Salmonella spp. and public health implications}

\section{Roberta Cristina da Rocha-e-Silva ${ }^{1 *}$, William Cardoso Maciel ${ }^{1}$, Régis Siqueira de Castro Teixeira ${ }^{2}$, Rosa Patrícia Ramos Salles ${ }^{3}$}

RESUMO: Os pombos domésticos e silvestres estão distribuídos por todo o mundo e carreiam micro-organismos patogênicos ao homem e a outros animais, podendo ser um dos responsáveis pela disseminação de Salmonella spp. Este patógeno gera grande preocupação para a economia mundial, uma vez que cria transtornos para a indústria avícola quando ocorre contaminação dos plantéis e ônus para a saúde pública devido a surtos de infecção alimentar causados por esta bactéria. Dessa forma, objetivou-se realizar um levantamento acerca da participaçáo do pombo doméstico na possível disseminação de Salmonella spp.

PALAVRAS-CHAVE: micro-organismos; disseminação; infecção alimentar.

\begin{abstract}
Domestic and wild pigeons are distributed throughout the world and carrie micro-organisms that are pathogenic to humans and other animals. They can be one of the animals in charge of the dissemination of Salmonella spp., pathogen of great concern for the world economy as it creates inconvenience to the poultry industry when there is contamination of herds and impacts on public health due to outbreaks of foodborne infection caused by this bacterium. Therefore, the objective was to conduct a survey about the participation of the domestic pigeon in the possible dissemination of Salmonella spp.
\end{abstract}

KEYWORDS: micro-organisms; dissemination; foodborne infection. 


\section{INTRODUÇÃO}

Os animais domésticos e silvestres são reservatórios de muitos micro-organismos patogênicos (Hinton; Bale, 1991), e seu contato próximo com os seres humanos pode representar risco à saúde pública (VÁzQUEz et al., 2010), pois desempenham um importante papel na epidemiologia de doenças entéricas em seres humanos (SKIRROw, 1991).

Diversas aves têm papel fundamental na contaminação de fontes de água potável e culturas agrícolas pelas fezes contaminadas, podendo transmitir agentes infecciosos a outras aves (Lillehaug et al., 2005), além de trazer riscos de contaminação para o homem e outros animais (Millán et al., 2004).

Dentre as espécies de aves, os pombos (Columba livia) aparecem como a principal ameaça para a saúde pública por serem reservatório de pelo menos 70 diferentes micro-organismos patogênicos para os humanos (HAAG-WACKERNAGEL; Moch, 2004), destacando-se a Salmonella enterica sorovar Typhimurium (Pedersen et al., 2006), sendo responsável por surtos em vários países, como Irlanda (Gorman; AdLEY, 2004), Noruega (Refsum et al., 2002), Espanha (Tirado et al., 2009), França (GAllay et al., 2000), entre outros.

Portanto, a revisão de literatura teve como objetivo realizar um levantamento acerca da participação do pombo doméstico na disseminação de Salmonella spp. e sua importância para a saúde pública.

\section{Desenvolvimento}

\section{Salmonelose}

O conhecimento do gênero Salmonella como agente patogênico com especificidade para as aves é datado de 1889 , quando Klein isolou Salmonella Gallinarum (BARrow, 1993) e Rettger descobriu, em 1899, S. Pullorum (Rettger, 1909). No entanto, a maioria dos sorovares de salmonela náo possui um hospedeiro específico, podendo acometer várias espécies distintas. As bactérias do gênero Salmonella são micro-organismos bacilares que medem aproximadamente 0,7 a 1,5 x 2,5 $\mu \mathrm{m}$. São gram-negativos e incapazes de esporular. $\mathrm{Na}$ maioria das vezes, são móveis, devido à presença de flagelos peritríquios, com exceção de $S$. Pullorum e $S$. Gallinarum, que não possuem flagelos e são imóveis (Holt et al., 1994).

Essas bactérias pertencem à família Enterobacteriaceae e compreendem um total de 2.579 sorotipos identificados, entre os quais 2.557 pertencem à espécie $S$. enterica e estão distribuídos entre as seis subespécies: $S$. enterica subsp. enterica (1.531); S. enterica subsp. salamae (505); S. enterica subsp. arizonae (99); S. enterica subsp. diarizonae (336); S. enterica subsp. houtenae (73); S. enterica subsp. indica (13).
Os demais sorovares (22) estão enquadrados na espécie S. bongori (SÁNCHEZ-VARGAS et al., 2011).

Nas aves, as salmonelas podem provocar três doenças distintas: a pulorose, por meio da infecção por $S$. enterica sorovar Pullorum; o tifo aviário, por $S$. enterica sorovar Gallinarum; e o paratifo aviário, causado por qualquer bactéria do gênero Salmonella, exceto os agentes da pulorose e do tifo aviário. Entre as salmonelas paratifoides, Salmonella Typhimurium, $S$. Agona e $S$. Enteritidis se destacam devido ao caráter zoonótico (SEO et al., 2000).

Salmonella spp. pode ser transmitida por via vertical, ou seja, da ave para o embrião, por meio da contaminação do ovo durante o desenvolvimento, ou após a passagem pela cloaca devido à presença de fezes (Olorunsola et al., 2012). A transmissão também pode ser por via horizontal, através do contato com aves doentes, seres humanos com fômites contaminados, equipamentos e água contendo o agente, além de aves silvestres, roedores (PAINTER et al., 2004), moscas (HolT et al., 2007) e ácaros contaminados (Moro et al., 2009). A principal porta de entrada é a via oral. Contudo, o agente patogênico pode penetrar no organismo hospedeiro pela via respiratória (TANNOCK; SMITH 1971), nasal, conjuntival, cloacal e umbilical (Cox et al., 1996).

Os ovos estão sujeitos à contaminação por Salmonella por meio da infecçáo do trato reprodutor antes da deposiçáo da casca, a partir da instalação do agente etiológico na gema ou no albúmen (SHIVAPRASAd et al., 1990), durante a passagem pela vagina (Мгумото et al., 1997) ou, ainda, pelas fezes, quando os micro-organismos penetram pela casca do ovo (De Reu et al., 2006).

Os sinais clínicos da salmonelose variam de acordo com a virulência da cepa e a suscetibilidade do animal. São observadas aves com penas eriçadas, asas caídas, dificuldade de respiração, diarreia (SHIVAPRASAD, 2000), redução da ingestão de alimentos, prostração, sonolência e olhos fechados (BERCHIERI Júnior; Freitas Neto, 2009).

O diagnóstico definitivo e eficaz é baseado no isolamento e na identificação do micro-organismo, principalmente em amostras de fezes por swab cloacal ou diretamente do reto do animal (НyATt; Weese, 2004) ou do ambiente (Окамото et al., 2009).

Associados ao exame microbiológico, podem ser realizados testes sorológicos de Ensaio de Imunoabsorção com Enzimas Ligadas (ELISA) (Oliveira et al., 2004), soroaglutinação rápida em placas (SANTos, 2009) e Reação de Cadeia em Polimerase (PCR) (Moussa et al., 2010).

\section{Pombo doméstico (Columba livia)}

Columba livia (pombo doméstico) é uma das 50 espécies pertencentes ao gênero Columba (NunEs, 2003). Essa ave é originária de países mediterrâneos, das costas e falésias da Europa, Norte da África e Ásia ocidental, e foi domesticada há cerca 
de 5 mil anos no Mediterrâneo Oriental (Levi, 1974), sendo introduzida na América do Norte, Central e do Sul e em toda a Europa (BAptista et al., 1997).

Os pombos estão presentes em áreas urbanizadas do mundo todo (Clergeau et al., 2006), convivendo junto à população humana (HaAG-WACKernagel, 2003). Devido à capacidade de voar longas distâncias, essas aves podem desempenhar um papel importante na epidemiologia de zoonoses (Fоті et al., 2009).

O tamanho da população de pombos é influenciado positivamente pelo número de habitantes humanos, pela área da cidade (Hetmański et al., 2010) e pelas características desta, como a idade dos edifícios (SACCHI et al., 2002), a distribuiçâo espacial dos recursos e o fornecimento de alimentos (Rose et al., 2006). A cidade de Barcelona possui uma das maiores densidades de aves em áreas urbanas do mundo (SENAR; Sol, 1991), ocasionando problemas significativos que geram altos custos, os quais são causados pelo excessivo número de pombos urbanos (ZucCONi et al., 2003). Nas cidades da província da Pomerânia, os pombos estão concentrados principalmente nos bairros centrais, em áreas com blocos de apartamentos e no subúrbio (HetMaŃski et al., 2010), enquanto em Amsterdã a maior concentração ocorre em áreas do setor público (Buiss; Van Wijnen, 2001).

No Japão, os pombos encontram-se amplamente distribuídos nas áreas urbana e rural, e entram em contato com os seres humanos em parques, templos, santuários, jardins públicos e estaçóes ferroviárias (TANAKA et al., 2005). Em Israel, a presença dessas aves tem sido relatada apenas em parques urbanos (Shwartz et al., 2008). Em alguns casos, os animais parecem ser atraídos para as áreas urbanizadas em busca de alimento e abrigo para construir seus ninhos (SwEeney et al., 1997), e a disponibilidade de fontes de alimento tem levado à proliferaçấo de pombos em algumas áreas urbanas (Sol, 2008).

Portanto, o grande fornecimento de alimento pela população, deliberada e acidentalmente, juntamente à ausência de predadores, permite o acúmulo de grandes populaçôes de pombos. Isso pode causar vários problemas (HaAG-WaCkernagel, 2003), uma vez que os pombos podem ser um reservatório de micro-organismos patogênicos para o homem (Buijs; VAN Wijnen, 2001), incluindo Salmonella spp. (HaAG-WaCKernagel; Мoch, 2004), agente responsável por surtos de infecção alimentar em todo o mundo.

Em 1933, foi registrado um surto de salmonelose humana ocasionado pela ingestâo de ovos de pombos que estavam contaminados com Salmonella enterica sorotipo Typhimurium, variante de Copenhagen (Clarenburg; Dornickx, 1933). No entanto, a disseminação de Salmonella sp. por pombos pode ocorrer também pela via horizontal, através das fezes. Foi relatado um caso de infecção em um homem causado por S. enterica sorovar Kiambu, após inalação do patógeno presente em fezes de pombos (LaCassin et al., 1995). A presença de Salmonella spp. com capacidade de infectar o homem foi relatada em várias análises de fezes de pombos de vida livre em diferentes países do mundo. Há registros de isolamento de Salmonella enterica sorotipo Typhimurium, patógeno responsável por inúmeros surtos de infecção alimentar humana desde a década de 1960 (Lee, 1974) na Bélgica (Pasmans et al., 2004), no Japão (TANAKa et al., 2005), na Croácia (Vucemilo et al., 2003), em Trinidad (Gopee et al., 2000) e em Barcelona (CASAnovas et al., 1995).

Em Grã Canária, na Espanha, foi relatado um surto por ingestáo de água potável contaminada com Salmonella Kottbus. Esta água era produzida e envazada em uma fábrica local, e nas suas proximidades observou-se a presença de pombos. Após coleta do material desses animais, constatou-se a presença de Salmonella spp., sugerindo assim que essas aves podem ter sido responsáveis pela contaminação da fonte de água (Palmera-Suárez et al., 2007). Portanto, a populaçấo de pombos no ambiente urbano é uma questão complexa, a qual demanda um planejamento cuidadoso das autoridades locais, de forma a evitar a superpopulaçáo dessas aves e, consequentemente, gastos dispendiosos com a saúde pública e o patrimônio. Mas antes de qualquer intervenção, é obrigatório realizar uma avaliação da situaçáo local quanto ao número de aves e seus locais de agregação (MAGNino et al., 2009), por isso é fundamental avaliar a eficácia das ações de controle (FEARE, 1991).

Salmonella da espécie bongori também já foram isoladas de fezes de pombos na Itália (Foti et al., 2009). Essa espécie é rara e nunca havia sido registrado como responsável por infecçôes em humanos ou animais, mas mostrou-se capaz de causar enterite aguda em crianças e, ocasionalmente, em imunodeficientes adultos e animais no sul da Itália (Giammanco et al., 2002).

\section{Controle de pombos em centros urbanos}

Muitas cidades tentam controlar a populaçáo de pombos por meio da captura seguida da eutanásia (KaUTZ; MALECKI, 1985), controle químico de natalidade e uma combinação de remoçáo de ovos em pombais, alimentaçáo e campanhas contra a matança (HAAG-WACKERNAGel, 2005).

A populaçáo de pombos pode ser controlada com a administraçấo de Nicarbazina (Ferri et al., 2009), um coccidiostático sintetizado quimicamente em laboratório que, quando administrado em aves, apresenta redução da eclodibilidade de ovos (Bynum et al., 2007). A administração de anticoncepcional também pode ser útil para reduzir a população de pássaros, bem como a proibiçáo de as pessoas alimentarem essas aves (Magnino et al., 2009). No entanto, esses métodos de controle são ineficazes, não apenas pelo efeito relativamente baixo na natalidade, mas também pela provável ausência da longa duração de efeitos. Isso pode levar 
a uma retomada rápida da atividade reprodutiva quando o tratamento for interrompido (Giunchi et al., 2007). $\mathrm{Na}$ Europa, a redução da população de pombos foi possível por meio do bloqueio das aberturas de edifícios (RAGNI et al., 1996) e controlando o fornecimento de alimentos para as aves (HAAG-WACKERNAGEL, 1993).

Portanto, devido a grande taxa de reprodução dos pombos, o controle só pode ser resolvido ao educar a população, para que esta não disponibilize alimentos a essas aves (HAAG-WACKERnAGEL, 2005), e restringindo locais de nidificação ou potenciais de abastecimento alimentar (SENAR, 2002).

\section{CONSIDERAÇÕES FINAIS}

Embora não existam muitos relatos de infecção alimentar em humanos ocasionados por pombos, são vários os registros acerca da presença de Salmonella spp. nessas aves, não podendo ser descartada a possibilidade da transmissão do patógeno ao ser humano e a outros animais, o que gera grandes transtornos na economia e na saúde pública. Vale ressaltar ainda a grande dificuldade em instituir medidas eficazes de controle dessas aves, o que poderia auxiliar a redução da possível disseminação de Salmonella spp.

\section{REFERÊNCIAS}

BAPTISTA, L.F.; TRAIL, P.W.; HORBLIT, H.M. Family Columbidae (Sandgrouse to Cuckoos). In: Handbook of birds of the world. Barcelona: Lynx Edicions. 1997. p.60-243.

BARROW, P.A. Salmonella - present, past and future. Avian Pathology, v.22, n.4, p.651-669, 1993.

BERCHIERI JÚNIOR, A.; FREITAS NETO, O.C. Salmoneloses. In: BERCHIERI JÚNIOR, A.; SILVA, E.N.; DI FÁBIO, J.; SESTI, L.; ZUANAZE, M.A.F. Doença das Aves. São Pauo: FACTA. 2009. p.435-454.

BUIJS, J.A.; VAN WIJNEN, J.H. Survey of feral rock doves (Columba livia) in Amsterdam, a bird-human association. Urban Ecosystems, v.5, n.4, p.235-241, 2001.

BYNUM, K.; EISEMANNY, J.D.; WEAVERZ, G.C.; YODER, C.A.; FAGERSTONEYY, K.A.; MILLER, L.A. Nicarbazin ovocontrol $G$ bait reduces hatchability of eggs laid by resident Canada geese in Oregon. Journal of Wildlife Management, v.71, n.1, p.135-143, 2007.

CASANOVAS, L.; SIMON, M.; FERRER, M.D.; ARQUES, J.; MONZONL, G. Intestinal carriage of Campylobacters, Salmonellas, Yersinias and Listerias in pigeons in the city of Barcelona. Journal of Applied Bacteriology, v.78, n.1, p.11-13, 1995.

CLARENBURG, A.; DORNICKX, C.G.J. Nahrungmittelvergiftung bei Menschen in Zusammenhang mit Tauben paratyphose. Zschr Hyg Inf Krankh, v.114, p.31-41, 1933.

CLERGEAU, P.; CROCI, S.; JOKIMAKI, J.; KAISANLAHTI-JOKIMAKI, M.L.; DINETTI, M. Avifauna homogenisation by urbanisation: analysis at different European latitudes. Biological Conservation, v. 127, n.3, p.336-344, 2006.

COX, N.A.; BAILEY, J.S.; BERRANG, M.E. Alternative routes for Salmonella intestinal tract colonization of chicks. Journal of Applied Poultry Research, v.5, n.3, p.282-288, 1996.

DE REU, K.; GRIJSPEERDT, K.; MESSENS, W.; HEYNDRICKW, M.; UYTTENDAELE, M.; DEBEVERE, J.; HERMAN, L. Eggshell factors influencing eggshell penetration and whole egg contamination by different bacteria, including Salmonella Enteritidis. International Journal of Food Microbiology, v. 1 12, n.3, p.253-260, 2006.
FEARE, C.J. Control of bird pest populations. In: Bird population studies. Oxford: Univ. 1991. p.463-478.

FERRI, M.; FERRARESI, M.; GELATI, A.; ZANNETTI, G.; UBALDI, A.; CONTIERO, B.; BURSI, E. Use of nicarbazinee in the control of urban pigeon colonies in Italy in 1990-2007. Annali della Facoltà di medicina Veterinaria di Parma, v.29, p.91-102, 2009.

FOTI, M.; DAIDONE, A.; ALEO, A.; PIZZIMENTI, A.; GIACOPELLO, C.; MAMMINA, C. Salmonella bongori 48:z35:- in Migratory Birds, Italy. Emerging Infectious Diseases, v. 15, n.3, p.502-503, 2009.

GALLAY, A.; VAILLANT, V.; BOUVET, P.; GRIMONT, P.; DESENCLOS, J.C. How many foodborne outbreaks of Salmonella infection occurred in France in 1995? American Journal of Epidemiology, v.152, n.2, p.171-177, 2000.

GIAMMANCO, G.M.; PIGNATO, S.; MAMMINA, C.; GRIMONT, F.; GRIMONT, P.A.D.; NASTASI, A.; GIAMMANCO, G. Persistent endemicity of Salmonella bongori 48:z35: in Southern Italy: molecular characterization of human, animal and environmental isolates. Journal of Clinic Microbiology, v.40, n.9, p.3502-3505, 2002.

GOPEE, N.V.; ADESIYUN, A.A.; CAESAR, K. Retrospective and longitudinal study of salmonellosis in captive wildlife in Trinidad. Journal of Wildlife Diseases, v.36, n.2, p.284-293, 2000.

GORMAN, R.; ADLEY, C.C. Characterization of Salmonella enterica serotype Typhimurium isolates from human, food, and animal sources in the Republic of Ireland. Journal of Clinical Microbiology, v.42, n.5, p.2314-2316, 2004.

GIUNCHI, D.; BALDACCINI, N.E.; SBRAGIA, G.; SOLDATINI, C. On the use of pharmacological sterilisation to control feral pigeon populations. Wildlife Reserch, v.34, n.4, p.306-318, 2007.

HAAG-WACKERNAGEL, D. Street pigeons in Base. Nature, p.361:200, 1993.

HAAG-WACKERNAGEL, D. Die Strassentaube: GeschichteProbleme - Lo sungen. DerOrnithologische Beobachter, v.100, p.33-57, 2003.

HAAG-WACKERNAGEL, D.; MOCH, H. Health hazards posed by feral pigeons. Journal of Infection, v.48, n.4, p.307-313, 2004. 
HAAG-WACKERNAGEL, D. Parasites from feral pigeons as a health hazard for humans. Annals of Applied Biology, v. 147, n.2, p.203-210, 2005.

HETMAŃSKI, T.; BOCHEŃSKI, M.; TRYJANOWSKI, P.; SKÓRKA, P. The effect of habitat and number of inhabitants on the population sizes of feral pigeons around towns in northern Poland. European Journal of Wildlife Research, v.57, n.3, p.421-428, 2010.

HINTON, M.; BALE, M.J. Bacterial pathogens in domesticated animals and their environment. Journal of Applied Bacteriology Symposium Supplement, v.70, p.81S-90S, 1991.

HOLT, P.S.; GEDEN, C.J.; MOORE, R.W.; GAST, R.K. Isolation of Salmonella enterica serovar Enteritidis from houseflies (Musca domestica) found in rooms containing Salmonella serovar Enteritidischallenged hens. Applied and Environmental Microbiology, v.73, n. 19, p.6030-6035, 2007.

HOLT, J.G.; KRIEG, N.R.; SNEATH, P.H.A.; STALEY, J.T.; WILLIAMS, S.T. Bergey's: manual of determinative bacteriology. Baltimore: Williams \& Wikins. 1994. p.186-187.

HYATT, D.R.; WEESE, J.S. Salmonella culture: sampling procedures and laboratory techniques. Veterinary Clinics of North America: Equine Practice, v.20, n.3, p.577-585, 2004.

KAUTZ, J.E.; MALECKI, R.A. Effects of harvest on feral pigeon survival, nest success and population size. International Fish and Wildlife Technical Report, v.31, n.p.1-16, 1985.

LACASSIN, F.; MINO, J.C.; BENOIT, C.; PERRONNE, C.; LEPORT, C.; VILDÉ, J.L. A propos d'un cas de salmonellose aviaire [A case of avian salmonellosis]. Revista Medicina Interna, v.16, p.77-78, 1995.

LEE, J.A. Recent trends in human salmonellosis in England and Wales: the epidemiology of prevalent serotypes other than Salmonella typhimurium. Journal of Hygiene, v.72, n.2, p.185-195, 1974.

LEVI, W. The Pigeon. Sumter, S.C.: Levi Publishing Co, Inc. 1974. p.4.

LILLEHAUG, A.; JONASSEN, C.M.; BERGSJØ, B.; HOFSHAGEN, M.; THARALDSEN, J.; NESSE, L.L.; HANDELAND, K. Screening of feral pigeon (Columba livia), mallard (Anas platyrhynchos) and graylag goose (Anser anser) populations for Campylobacter spp., Salmonella spp., Avian Influenza Virus and Avian Paramyxovirus. Acta Veterinaria Scandinavica, v.46, n.4, p. 193-202, 2005.

MAGNINO, S.; HAAG-WACKERNAGEL, D.; GEIGENFEIND, I.; HELMECKE, S.; DOVC, A.; PRUKNER-RADOVCIC, E.; RESIDBEGOVIC, E.; ILIESKIG, V.; LAROUCAU, K.; DONATI, M.; MARTINOV, S.; KALETA, E.F. Chlamydial infections in feral pigeons in Europe: Review of data and focus on public health implications. Veterinary Microbiology, v.135, n.1-2, p.54-67, 2009.

MILLÁN, J.; ADURIZ, G.; MORENO, B.; JUSTE, R.A.; BARRAL, M. Salmonella isolates from wild birds and mammals in the Basque Coutry (Spain). Scientific and Techical Review, v.23, n.3, p.905-91 1, 2004.

MIYAMOTO, T.; BABA, E.; TANAKA, T.; SASAI, K.; FUKATA, T.; ARAKAWA, A. Salmonella Enteritidis contamination of eggs from hens inoculated by vaginal, cloacal and intravenous routes. Avian Diseases, v.41, n.2, p.296-303, 1997.
MORO, C.V.; DE LUNA, C.J.; TOD, A.; GUY, J.H.; SPARAGANO, O.A.; ZENNER, L. The poultry red mite (Dermanyssus gallinae): a potential vector of pathogenic agents. Experimental and Applied Acarology, v.48, n.1-2, p.93-104, 2009.

MOUSSA, I.M.; GASSEM, M.A.; AL-DOSS, A.A.; MAHMOUD, W.A.S.; ABDEL MAWGOOD, A.L. Using molecular techniques for rapid detection of Salmonella serovars in frozen chicken and chicken products collected from Riyadh, Saudi Arabia. Journal of Biotechnology, v.9, n.5, p.612-619, 2010.

NUNES, V.F.P. Pombos urbanos: 0 desafio de controle. Arquivos do Instituto Biológico, São Paulo, v.65, n. 1, p.89-92, 2003.

OLIVEIRA, G.H.; BERCHIERI JR., A.; MONTASSIER, H.J.; FERNANDES, A.C. Assessment of sorological response of chickens to Salmonella Gallinarum and Salmonella Pullorum by ELISA. Revista Brasileira de Ciência Avícola, v.6, n.2, p.111-115, 2004.

OLORUNSOLA, R.A.; ERUVBETINE, D.; OYEKUNLE, A.M.; OGUNADE, I.M. Salmonella organism transmission in hatching broiler eggs. Journal of Biology, Agriculture and Healthcare, v.2, n.10, p.13-16, 2012.

PAINTER, J.A.; MøLBAK, K.; SONNE-HANSEN, J.; BARRETT, T.; WELLS, J.G.; TAUXE, R.V. Salmonella-based rodenticides and public health. Emerging Infectious Diseases, v.10, n.6, p.985, 2004.

PALMERA-SUÁREZ, R.; GARCÍA, P.; GARCÍA, A.; BARRASA, A.; HERRERA, D. Salmonella Kottbus outbreak in infants in Gran Canaria (Spain), caused by bottled water, August-November 2006. Euro Surveillance, v.12, n.7, p.3235, 2007.

PASMANS, F.; VAN IMMERSEEL, F.; HERMANS, K.; HEYNDRICKX, M.; COLLARD, J.M.; DUCATELLE, R.; HAESEBROUCK, F. Assessment of virulence of pigeon isolates of Salmonella enterica subsp. enterica serovar Typhimurium variant Copenhagen for Humans. Journal of Clinical Microbiology, v.42, n.5, p.2000-2002, 2004.

PEDERSEN, K.; CLARK, L.; ANDELT, W.F.; SALMAN, M.D. Prevalence of Shiga toxin-producing Escherichia coli and Salmonella enterica in rock pigeons captured in fort collins, Colorado. Journal of Wildlife Diseases, v.42, n. 1, p.46-55, 2006.

RAGNI, B.; VELATTA, F.; MONTEFAMEGLIO, M. Restrizione dell'habitat per il controllo della popolazione urbana di Columba livia. In: Control of Synanthropic bird populations: problems and prospectives. Roma: WHO/FAO. 1996. p.106-110.

REFSUM, T.; HANDELAND, K.; BAGGESEN, D.L.; HOLSTAD, G.; KAPPERUD, G. Salmonellae in avian wildlife in Norway from 1969 to 2000. Applied and Environmental Microbiology, v.68, n. 11 , p.5595-5599, 2002.

RETTGER, L.F. Further studies on fatal septicemia in young chickens, or "white diarrhea". The Journal of medical research, v.21, n. 1, p. $115,1909$.

ROSE, E.; NAGEL, P.; HAAG-WACKERNAGEL, D. Spatio-temporal use of the urban habitat by feral pigeons (Columba livia). Behavioral Ecology and Sociobiology, v.60, n.2, p.242-254, 2006.

SACCHI, R.; GENTILLI, A.; RAZZETTI, E.; BARBIERI, F. Effects of building features on density and flock distribution of feral pigeons Columba livia var. domestica in an urban environment. Canadian Journal of Zoology, v.80, n. 1, p.48-54, 2002. 
SENAR, J.C. Fauna urbana: ¿control o gestión? La Tierra, p.48:33, 2002.

SENAR, J.C.; SOL, D. Censo de palomas Columba livia var. de la ciudad de Barcelona: Aplicación del muestreo estratificado con factor de corrección. Butlletí del Grup Catalá d'Anellament, v.8 p.19-24, 1991.

SÁNCHEZ-VARGAS, F.M.; ABU-EL-HAIJA, M.A.; GÓMEZDUARTE, O.G. Salmonella infections: an update on epidemiology, management, and prevention. Travel Medicine and Infectious Disease, v.9, n.6, p.263-277, 2011.

SANTOS, C.H.C. Diagnóstico microbiológico e sorológico. In BERCHIERI JR., A.; SILVA, E.N.; DI FÁBIO, J.; SESTI, L.; ZUANAZE, M.A.F., Doença das Aves. São Paulo: FACTA. 2009, p.79-104.

SEO, K.H.; HOLT, P.S.; GAST, R.K.; HOFACRE, C.L. Elimination of early Salmonella Enteritidis infection after treatment with competitive-exclusion culture and enrofloxacin in experimentally infected chicks. Poultry Science, v.79, n. 10, p. 1408-1413, 2000.

SHIVAPRASAD, H.I. Fowl typhoid and pullorum disease. Revue Scientifique et Technique, v.19, n.2, p.405-424, 2000.

SHIVAPRASAD, H.L.; TIMONEY, J.F.; MORALES, S.; LUCIO, B.; BAKER, R.C. Pathogenesis of Salmonella Enteritidis infection in laying chickens. i. studies on egg transmission, clinical signs, fecal shedding, and serological responses. Avian Diseases, v.34, n.3, p.548-557, 1990.

SHWARTZ, A.; SHIRLEY, S.; KARK, S. How do habitat variability and management regime shape the spatial heterogeneity of birds within a large Mediterranean urban park? Landscape and Urban Planning, v.84, n.3-4, p.219-229, 2008.

SKIRROW, M.B. Epidemiology of Campylobacter enteritis. International Journal of Food Microbiology, v. 12, n. 1, p.9-16, 1991.
SOL, D. Artificial selection, naturalization, and fitness: Darwin's pigeons revisited. Biological Journal of the Linnean Society, v.93, n.4, p.657-665, 2008.

SWEENEY, S.J.; REDIG, P.T.; TORDOFF, H.B. Morbidity, survival and productivity of rehabilitated Peregrine Falcons in upper midwest. U.S. Journal of Raptor Research, v.31, n.4, p.347-352, 1997.

TANAKA, C.; MIYAZAWA, T.; WATARI, M.; ISHGURO, N. Bacteriological survey of feces from feral pigeon in Japan. Journal of Veterinary Medical Science, v.67, n.9, p.951-953, 2005.

TANNOCK, G.W.; SMITH, J.M.B. A Salmonella carrier state involving the upper respiratory tract of mice. Journal of Infection Disease, v.123, n.4, p.502-506, 1971.

TIRADO, M.D.B.; ROSARIO, M.M.; CELADES, M.E.P.; BELLIDOBLASCO, J.; PARDO F.J.S. Evolución de los serotipos, fagotipos y resistencia a antimicrobianos de Salmonella sp. en el departamento de salud 02 de la provincia de Castellón, España (2000-2006). Revista Chilena de Infectologia, v.26, n.6, p.520-527, 2009.

VÁZQUEZ, B.; ESPERÓN, F.; NEVES, E.; LÓPEZ, J.; BALLESTEROS, C.; MUÑOZ, M.J. Screening for several potential pathogens in feral pigeons (Columba livia) in Madrid. Acta Veterinaria Scandinavica, v.52, n.45, p. 1-6, 2010.

VUCEMILO, M.; VLAHOVIC, K.; DOVC, A.; MUZINIC, J.; PAVLAIG, M.; JERCIC, J.; ZUPANCIC, Z. Prevalence of Campylobacter jejuni, Salmonella Typhimurium, and avian Paramyxovirus type 1 (PMV-1) in pigeons from different regions in Croatia. Zeitschrift für Jagdwissenschaft, v.49, n.4, p.303-313, 2003.

ZUCCONI, S.; GALAVOTTI, S.; DESERTI, R. I colombi in ambiente urbano. Sintesi del progetto di ricerca Nomisma. Igiene Alimenti. Disinfestazione \& Igiene Ambientale, 9-22, 2003. 